\title{
Crystal structure of $8 \mathrm{a}, 8 \mathrm{a}^{\prime \prime}$-oxybis(8aH-8,9-dioxa- $3 a^{1} \lambda^{4}$-aza-8a $\lambda^{4}$-borabenzo[fg]tetracene), $\mathrm{C}_{34} \mathrm{H}_{22} \mathrm{~B}_{2} \mathrm{~N}_{2} \mathrm{O}_{5}$
}

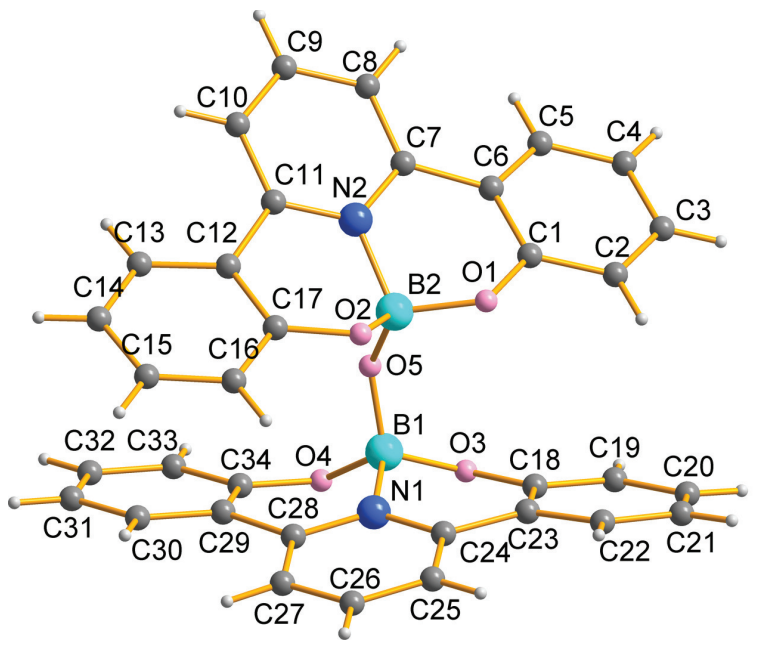

https://doi.org/10.1515/ncrs-2020-0136

Received March 10, 2020; accepted April 6, 2020; available online April 24, 2020

\begin{abstract}
$\mathrm{C}_{34} \mathrm{H}_{22} \mathrm{~B}_{2} \mathrm{~N}_{2} \mathrm{O}_{5}$, monoclinic, $P 2_{1} / c$ (no. 14), $a=9.9886(3) \AA$, $b=17.8995(6) \AA, \quad c=14.3437(5) \AA, \quad \beta=98.141(3)^{\circ}$, $V=2538.68(15) \AA^{3}, Z=4, R_{\mathrm{gt}}(F)=0.0391, w R_{\mathrm{ref}}\left(F^{2}\right)=0.1022$, $T=100 \mathrm{~K}$.
\end{abstract}

\section{CCDC no.: 1932230}

Table 1 contains crystallographic data and Table 2 contains the list of the atoms including atomic coordinates and displacement parameters.

\section{Source of material}

The title complex was synthesized from a mixture of 2,6-bis(2methoxyphenyl)pyridine $(0.5 \mathrm{mmol})$ which was dissolved

\footnotetext{
*Corresponding author: Zongcheng Miao, Key Laboratory of Organic Polymer Photoelectric Materials, School of Science, Xijing University, Xi'an 710123, Shaanxi Province, P.R. China, e-mail: miaozongcheng@xijing.edu.cn

Siyi Ding, Qiang Ma and Huaping Ren: Key Laboratory of Organic Polymer Photoelectric Materials, School of Science, Xijing University, Xi'an 710123, Shaanxi Province, P.R. China. https://orcid.org/0000-0001-5446-0254 (S. Ding)
}

๑ Open Access. ( 2020 Siyi Ding et al., published by De Gruyter. (cc) BY License.
Table 1: Data collection and handling.

\begin{tabular}{ll}
\hline Crystal: & Yellow needle \\
Size: & $0.31 \times 0.09 \times 0.06 \mathrm{~mm}$ \\
Wavelength: & Mo $K \alpha$ radiation $(0.71073 \AA)$ \\
$\mu:$ & $0.10 \mathrm{~mm}^{-1}$ \\
Diffractometer, scan mode: & APEX2, $\varphi$ and $\omega$ \\
$\theta_{\max }$, completeness: & $25.0^{\circ},>99 \%$ \\
$N(h k l)_{\text {measured }}, N(h k l)_{\text {unique }}, R_{\text {int }}:$ & $13538,4470,0.039$ \\
Criterion for $I_{\text {obs }}, N\left(h k l_{\text {gt }}:\right.$ & $I_{\text {obs }}>2 \sigma\left(l_{\text {obs }}\right), 3245$ \\
$N(\text { param })_{\text {refined }}:$ & 388 \\
Programs: & SHELX [1-3], Bruker [4] \\
\hline
\end{tabular}

in anhydrous DCM $(10 \mathrm{~mL})$, then cooled to $-78^{\circ} \mathrm{C}, \mathrm{BBr}_{3}$ (4 mmol) in anhydrous DCM was added slowly. The mixture was stirred until room temperature was reached. The yellow solid was filtered under a nitrogen atmosphere, the filter cake was washed using anhydrous hexane.

\section{Experimental details}

All the $\mathrm{H}$ atoms on the benzene rings were placed geometrically and refined without any constraints or restraints.

\section{Comment}

Phenol-pyridyl boron complexes, which have polydentate chelating sites, are one of the hotspots of coordination chemists. They are versatile building blocks in functional molecules, therefore, new methods for their preparation have attracted interests. Among this large family the well-known members are 1,6-bis(2-hydroxy-5methylphenyl)pyridine ((mdppy)BF) [5, 6] and 1,6-bis(2hydroxy-5-methylphenyl)pyridylboron substituted benzene $[7,8]$, which are used in OLEDS and fluoride ion sensors [58]. The change of functional groups attached to the boron atom have a great influence on their activitites $[9,10]$.

In the asymmetric unit, there is only one molecule (see the Figure). The electron-deficient boron atom has the ability to reach the quartet coordination, meanwhile the nitrogen atom can offer its lone pair electrons to boron forming a coordination bond. In this compound $\mathrm{B} 1,03,04,05$ and the N1 constitute a distorted tetrahedral configuration, together with another distorted tetrahedral configuration consisting of 
Table 2: Fractional atomic coordinates and isotropic or equivalent isotropic displacement parameters $\left(\AA^{2}\right)$.

\begin{tabular}{|c|c|c|c|c|}
\hline Atom & $x$ & $y$ & $z$ & $U_{\text {iso }} * / U_{\text {eq }}$ \\
\hline B1 & $-0.0857(2)$ & $0.14033(12)$ & $0.72871(14)$ & $0.0142(5)$ \\
\hline B2 & $0.0903(2)$ & $.03227(12)$ & $.73065(14)$ & $.0145(5)$ \\
\hline C1 & $-0.05387(18)$ & $-0.07591(10)$ & 1(13) & $0177(4)$ \\
\hline$C 2$ & $-0.16145(19)$ & $-0.11201(11)$ & $0.74463(14)$ & $0227(5)$ \\
\hline $\mathrm{H} 2$ & -0.178975 & -0.103755 & 0.807198 & $0.027^{\star}$ \\
\hline$C 3$ & $-0.2430(2)$ & $-0.15987(11)$ & $0.68615(16)$ & $0.0290(5)$ \\
\hline 13 & -0.315694 & -0.184836 & 0.709039 & $0.035^{\star}$ \\
\hline-4 & $-0.2195(2)$ & $-0.17185(12)$ & $.59394(16)$ & $.0294(5)$ \\
\hline H4 & -0.277218 & -0.203878 & 0.553515 & $0.035^{*}$ \\
\hline 5 & $-0.1121(2)$ & $-0.13697(11)$ & $.56175(15)$ & $.0256(5)$ \\
\hline 5 & -0.095987 & -0.145414 & 0.498902 & $0.031^{*}$ \\
\hline 6 & $-0.02587(19)$ & $-0.08906(10)$ & $0.62023(13)$ & $0.0191(4)$ \\
\hline$C 7$ & $0.09438(19)$ & -0.0 & $0.58878(13)$ & $0.0185(4)$ \\
\hline 8 & $0.1477(2)$ & $5(11)$ & (13) & $0.0222(5)$ \\
\hline H8 & 0.105811 & -0.119634 & 717 & $0.027^{\star}$ \\
\hline 9 & $0.2613(2)$ & -0.046 & 13) & $0245(5)$ \\
\hline H9 & 0.296875 & -0.0 & 02 & 029* \\
\hline 10 & 0.3 & 0.0 & 3) & $6(5)$ \\
\hline 10 & 0.40 & 3452 & 749 & $0.026^{*}$ \\
\hline 11 & 18) & 11) & 12) & $5(4)$ \\
\hline 12 & $0.32842(18)$ & $0.09426(10)$ & 0.681 & $0.0169(4)$ \\
\hline 13 & 0.424 & & & $7(5)$ \\
\hline 113 & 0.4 & 622 & 169 & $0.025^{\star}$ \\
\hline 14 & 490 & & 4) & \\
\hline 14 & 0.5 & & 86 & $28^{*}$ \\
\hline 15 & 463 & 11) & 14) & 19(5) \\
\hline 15 & 0.5 & & & $0.026^{*}$ \\
\hline 16 & 3671 & 10) & 13) & $0180(4)$ \\
\hline 16 & 0.34 & & & $0.022^{\star}$ \\
\hline 17 & 0.2968 & & & 49(4) \\
\hline 18 & $-0.23826(19)$ & 0.072 & 13) & $0.0169(4)$ \\
\hline 19 & $-0.35159(19)$ & & 4) & $25(5)$ \\
\hline H19 & -0.412361 & 81 & 18 & $.027^{\star}$ \\
\hline 20 & $-0.3764(2)$ & 396(11) & 14) & $.0264(5)$ \\
\hline-120 & -0.455250 & -0.044092 & 25 & $.032 *$ \\
\hline 21 & $-0.2865(2)$ & -0.011 & .9707 & $0263(5)$ \\
\hline-121 & -0.301636 & -0.040888 & 1.022999 & $0.032^{\star}$ \\
\hline 0 & -0.1 & 0.03 & 0.977 & $0.0225(5)$ \\
\hline $\mathrm{H} 22$ & -0.114336 & 0.037166 & 1.034241 & $0.027^{\star}$ \\
\hline C23 & -0.15100 & & 3) & $0.0173(4)$ \\
\hline$C 24$ & $-0.04681(18)$ & $0.13796(10)$ & 0.9103 & $0.0170(4)$ \\
\hline & $0.01972(19)$ & $0.16199(12)$ & $0.99706(13)$ & $0.0225(5)$ \\
\hline-125 & 0.003340 & 0.137847 & 1.053340 & $0.027^{\star}$ \\
\hline C26 & .10904(19) & $22062(12)$ & $.00112(14)$ & $.0242(5)$ \\
\hline $\mathrm{H} 26$ & 0.15 & 6583 & 1.060191 & $0.029^{\star}$ \\
\hline C27 & $0.13159(19)$ & $0.25632(11)$ & $0.91972(13)$ & $0.0212(5)$ \\
\hline $\mathrm{H} 27$ & & & 91 & $0.025^{\star}$ \\
\hline C28 & $0.06700(18)$ & $0.23133(10)$ & $0.83291(13)$ & $0.0166(4)$ \\
\hline $\mathrm{C} 2$ & 0.085 & 0.265 & $0.74273(13)$ & $0.0162(4)$ \\
\hline C30 & $0.19096(19)$ & $0.31566(11)$ & $0.73481(14)$ & $0.0214(5)$ \\
\hline $\mathrm{H}_{3}$ & 0.255144 & 0.325982 & 0.788708 & $0.026^{*}$ \\
\hline C31 & $0.2041(2)$ & $0.35021(11)$ & $0.65087(14)$ & $0.0235(5)$ \\
\hline 12 & 0.276722 & 0.383830 & 0.647047 & $0.028^{\star}$ \\
\hline C32 & $0.11050(19)$ & $0.33558(11)$ & $0.57178(14)$ & $0.0218(5)$ \\
\hline
\end{tabular}

Table 2 (continued)

\begin{tabular}{lrrrr}
\hline Atom & $\boldsymbol{x}$ & $\boldsymbol{y}$ & $\boldsymbol{z}$ & $\boldsymbol{U}_{\text {iso }} \boldsymbol{U}_{\text {eq }}$ \\
\hline H32 & 0.119079 & 0.359326 & 0.513682 & $0.026^{*}$ \\
C33 & $0.00488(19)$ & $0.28659(10)$ & $0.57730(13)$ & $0.0183(4)$ \\
H33 & -0.059176 & 0.276997 & 0.523093 & $0.022^{*}$ \\
C34 & $-0.00784(17)$ & $0.25133(10)$ & $0.66197(13)$ & $0.0149(4)$ \\
N1 & $-0.02051(14)$ & $0.17233(9)$ & $0.83027(10)$ & $0.0153(4)$ \\
N2 & $0.15603(14)$ & $0.00083(8)$ & $0.64192(10)$ & $0.0154(4)$ \\
O1 & $0.02476(12)$ & $-0.03014(7)$ & $0.77155(8)$ & $0.0173(3)$ \\
O2 & $0.20188(12)$ & $0.05422(7)$ & $0.80228(8)$ & $0.0154(3)$ \\
O3 & $-0.21778(12)$ & $0.11018(7)$ & $0.73759(8)$ & $0.0183(3)$ \\
O4 & $-0.11339(12)$ & $0.20475(7)$ & $0.66575(8)$ & $0.0149(3)$ \\
O5 & $0.00512(12)$ & $0.09099(7)$ & $0.69360(8)$ & $0.0149(3)$ \\
\hline
\end{tabular}

B2, O1, O2, O5 and N2. All bonds and angles in the crystal structure are within the normal range.

Acknowledgements: This project was funded by the Scientific Research Program Funded by Shaanxi Provincial Education Department (Program No.18JK1194) and Scientific Research Program Funded by Shaanxi Provincial Education Department (Program No.18JK1204), China.

\section{References}

1. Sheldrick, G. M.: A short history of SHELX. Acta Crystallogr. A64 (2008) 112-122.

2. Sheldrick, G. M.: SHELXT - Integrated space-group and crystal-structure determination. Acta Crystallogr. A71 (2015) 3-8.

3. Sheldrick, G. M.: Crystal structure refinement with SHELXL. Acta Crystallogr. C71 (2015) 3-8.

4. Bruker. APEX2. SAINT and SADABS. Bruker AXS Inc, Madison, WI, USA (2009).

5. Liu, Y.; Guo, J.-H.; Zhang, H.-D.; Wang, Y.: Highly efficient white organic electroluminescence from a double-layer device based on a boron hydroxyphenylpyridine complex. Angew. Chem. Int. Ed. 41 (2002) 182-184.

6. Li, Y.-Q.; Liu, Y.; Bu, W.-M.; Guo, J.-H.; Wang, Y.: A mixed pyridine-phenol complex as an organic electroluminescent material. Chem. Commun. 16 (2000) 1551-1552.

7. Wang, H.-L.; Wang, X.-P.; Zhang, C.-L.: Theoretical study on electronic structures and luminescent properties of phenolpyridyl boron complexes. J. Mol. Struct.: THEOCHEM 819 (2007) 60-65.

8. Zhang, H.-Y.; Huo, C.; Ye, K.-Q.; Zhang, P.; Tian, W.-J.; Wang, Y.: Synthesis, structures, and luminescent properties of phenolpyridyl boron complexes. Inorg. Chem. 45 (2006) 2788-2794.

9. Lu, D.-M.; Wu, C.; Li, P.-F.: Synergistic effect of lewis bases and substituents on the electronic structure and reactivity of boryl radicals. Chem. Eur. J. 20 (2014) 1630-1637.

10. Lu, D.-M.; Wu, C.; Li, P.-F.: 3-Center-5-electron boryl radical with $\sigma^{0} \pi^{1}$ ground state electronic structure. Org. Lett. 16 (2014) 1486-1489. 